\title{
Convergence in the ERM and Declining Numbers of Common Stochastic Trends
}

\author{
Jesper Rangvid* and Carsten Sørensen \\ Department of Finance, Copenhagen Business School
}

November 2000

Keywords: $\quad$ Target zones, shadow exchange rates, convergence, recursive and rolling cointegration tests

JEL-classification: $\quad$ F31, F33, G15

${ }^{*}$ Corresponding author. Address: Solbjerg Plads 3, 5th floor, DK-2000 Frederiksberg, Denmark. Phone: (45) 3815 3615, fax: (45) 3815 3600, and e-mail: jr.fi@cbs.dk.

This article has benefitted from the comments of Tom Engsted, Jan Jakobsen, Henrik Jensen, and Piet Sercu and from seminar participants at Copenhagen Business School, the Zeuthen Workshop on International Macroeconomics, the European Finance Association's meeting in Helsinki (August 1999), the Royal Economic Association's meeting in St. Andrews (July 2000), and the World Congress of the Econometric Society in Seattle (August 2000). Gabriele Becker from the Bank of International Settlements as well as Heino Bohn and Niels Lynggård Hansen from Danmarks Nationalbank provided us with the data used in the paper. 


\begin{abstract}
In this paper, we use recursive and rolling cointegration methods to test for a system of several exchange rates being within the process of convergence. We use the methods to analyze how the convergence of five ERM exchange rates has developed during the ERM period. We find that the number of cointegration vectors in the system of ERM exchange rates increases as the sample period is extended, and interpret this as a sign of increased convergence of ERM exchange rates. In particular, we find no evidence of convergence in the first years of the ERM and strong evidence of convergence in the last years of the ERM. In the analyses we acknowledge that managed exchange rates, such as exchange rates in ERM target zones, can be misaligned at their observed values as compared to their fundamental free-float values. For this reason, we also study convergence of filtered shadow exchange rates. We use two filters to extract the shadow exchange rates: a linear filter and a non-linear filter.
\end{abstract}




\section{Introduction}

On January 1, 1999 eleven former ERM (European Exchange Rate Mechanism) currencies were replaced with the euro. In order to pave the way for a smooth transition to a common currency, the economies of the participating countries had to fulfill the convergence criteria of the Maastricht Treaty. The possible convergence of the ERM economies during the period leading up to the third stage of Economic and Monetary Union in Europe has prompted a considerable literature. ${ }^{1}$ This paper aims at contributing to this literature by $(i)$ presenting and discussing how specific cointegration procedures that allow for time-variation in the data-generating process can be used to investigate whether a system of non-converged exchange rates evolves into a system of converged exchange rates and (ii) use the procedures to test for convergence of ERM exchange rates.

It has been argued that a necessary condition for several integrated and nonstationary time series having already converged, is that the means and variances of suitable linear combinations of the time series (for instance the difference between two time series) do not drift apart in an unbounded fashion; in other words, the time series should be cointegrated, see Bernard \& Durlauf (1995, 1996) and Hall et al. (1992, 1997). Therefore, if the aim of an analysis is to investigate whether the particular time series included in the analysis have already converged, one of the more standard cointegration techniques can be applied. If, on the other hand, the aim of an analysis is to test for several time series being within the process of converging, tests which do not allow for time variation in the underlying data-generating process could be biased towards rejecting convergence; see also the discussion in Durlauf \& Quah (1999) on this point. When testing for the relevant time series being within the process of converging, it thus appears preferable to use methods which explicitly take into account the possible changes in the parameters of the underlying statistical model which could result from a process of convergence. For this reason, we propose to use time-varying multivariate cointegration techniques when analyzing the dynamics of convergence.

Especially, we use recursive cointegration tests, as presented in Hansen \& Johansen $(1992,1998)$, as well as rolling cointegration tests. We use the recursive tests to study the dynamics of convergence for the full sample of observations, whereas the rolling tests are used to investigate the degree of convergence during different subsamples of the full

\footnotetext{
${ }^{1}$ Surveys can be found in Kenen (1995), Eichengreen (1997), and Gros \& Thygesen (1998).
} 
sample. ${ }^{2}$ Basically, our hypothesis will be that when the time series start to converge, the probability of rejecting the above mentioned necessary condition for convergence (cointegration of the time series) should decline. In other words: the more converged the exchange rates are, the higher the chance of accepting that the system of exchange rates is characterized by relatively more cointegration vectors and thus relatively fewer "common" stochastic trends. ${ }^{3}$

In the empirical application of this paper, we illustrate the procedure with the ERM case and start the estimations in 1979 where the ERM was launched. Especially, we investigate whether the number of common stochastic trends decreased as spring 1998 approached (where the number of currencies to be replaced with the euro was finally laid down).

By employing multivariate cointegration techniques to study certain time-series properties of systems including several exchange rates, the paper is related to the analysis in Baillie \& Bollerslev (1989, 1994) and Diebold et al. (1994) who investigate issues related to the efficiency of foreign exchange markets by evaluating whether several floating exchange rates share common stochastic trends. Before the transition to a common currency, though, the exchange rates we analyze were not floating, but were de facto restricted by the ERM target zone regimes. This implies that if convergence is measured by means of bilateral ERM exchange rates only, the results could be biased towards a more favorable degree of convergence if the exchange rates were misaligned at their observed values, in the non-credible target zones, as compared to their fundamental free-float values. ${ }^{4}$ We take this aspect into account by recognizing that a positive spread between a domestic and a foreign interest rate could reflect that the specific exchange rate is temporarily misaligned at its observed value. Therefore, our analysis

\footnotetext{
${ }^{2}$ Furthermore, there is an additional statistical reason for using both types of time-varying tests: when doing the recursive tests, the number of observations will be increasing, whereas the number of observations is kept fixed in each rolling estimation. For this reason, the power of the tests remains constant in each rolling estimation.

${ }^{3}$ Generally, we think of a convergence process as a gradual and smooth process. For this reason, econometric techniques that test for structural breaks in the data-generating process at well-defined points in time (such as for instance the Perron, 1989, univariate tests for unit roots) are ill-suited for analyzing the kind of questions raised in this paper. In order to analyze how the process evolves over time, one needs techniques that allow for an investigation of the gradual change in the data-generating process, such as rolling and recursive techniques.

${ }^{4}$ As the ERM target zones could be realigned, they were non-credible target zones in the sense of Krugman (1991). Therefore, a pressure for a realignment could be realized whenever an exchange rate in the target zone system was misaligned relative to its fundamental free-float value.
} 
of convergence is based both on the actual exchange rates, as well as filtered shadow exchange rates, where the shadow exchange rates can be thought of as the natural levels of the exchange rates if the ERM target zones were suspended. Specifically, we use two procedures to filter out the shadow exchange rates: a linear filter and a non-linear filter, where the latter is taken from Rangvid \& Sørensen (2000) who present and estimate a model that describes the dynamics of an exchange rate in a non-credible target zone regime.

The basic result of the analysis is that convergence of ERM exchange rates has increased over time. We draw this conclusion because both the recursive tests and the rolling tests indicate that the system of ERM exchange rates is driven by relatively fewer common stochastic trends in the later parts of the sample period as compared to the early parts of the sample period. Furthermore, the rolling estimates allow us to infer that convergence was "strongest" during the period from 1995 to 1997 and "weakest" during the period from 1979 to 1983. Finally, we find that not all exchange rates are stationary when evaluated over the complete sample period from 1979 to 1998, but, importantly, all exchange rates are found to be stationary when evaluated over the latest subsample which runs from 1995 through 1998. In addition to these findings, which all are based on the estimations of multivariate models, we also employ traditional univariate Dickey-Fuller tests to find that the individual exchange rates in our sample behaved in an increasingly stationary manner as the ERM evolved. It is noted that the findings all are robust in the sense that they hold regardless of whether convergence is tested with actually observed exchange rates or with filtered shadow exchange rates.

In the following section, we present the statistical tests for the number of common stochastic trends, and we discuss the relation between, on the one side, the dynamics in the number of common stochastic trends that is found and, on the other side, convergence in the ERM. In section 3, we discuss the data which is analyzed in the empirical part of the paper and we describe how we have filtered out the shadow exchange rates. Section 4 contains the empirical part of the paper. In this section we present the empirical applications with the recursive and rolling tests for the number of common stochastic trends driving the ERM (shadow) exchange rates. A final section concludes. 


\section{Testing for convergence. The statistical procedure}

All tests employed in this paper basically evaluate whether the individual non-stationary time series in a multivariate system are driven by a reduced number of common stochastic trends. In the cointegration/common trends framework, the presence of $r$ cointegration vectors in a multivariate time series of dimension $p$ implies that there are $r$ relations that connect the $p$ variables linearly, i.e. there will only be $p-r=n$ independent non-stationary time series - the common stochastic trends. When testing for a decreasing number of common stochastic trends, or equivalently an increasing number of cointegration vectors, we apply recursive cointegration tests based on the Johansen procedure, see e.g. Johansen (1988, 1991, 1995), and, in the following, we briefly illustrate this testing procedure.

Consider the vector-autoregressive model $(V A R)$ written in the error correction form,

$$
\Delta Y_{t}=\sum_{i=1}^{k-1} \Gamma_{i} \Delta Y_{t-i}+\Pi Y_{t-1}+\varepsilon_{t}
$$

where $Y_{t}$ is of dimension $p$. With the model given by (1), the first part of Granger's representation theorem (see Johansen, 1991) states that if $\Pi$ has reduced rank $r<p$, the series in $Y_{t}$ contain unit roots, are cointegrated, and $\Pi$ can be decomposed as $\alpha \beta^{\prime}$, where both $\alpha$ and $\beta$ have dimensions $p \times r$. When $Y_{t}$ is integrated of order one, $\beta^{\prime} Y_{t}$ gives the stationary linear combinations of the otherwise non-stationary variables contained in $Y_{t}$, i.e. $\beta$ contains the $r$ cointegration vectors. The parameters in $\alpha$ are often referred to as the loading coefficients.

\subsection{The relation between cointegration and common trends}

The second part of Granger's representation theorem provides the $M A$-representation of a cointegrated multivariate process. To be more specific, the $M A$-representation of $\Delta Y_{t}$ is given by $(1-L) Y_{t}=C(L) \varepsilon_{t}$, with $L$ as the lag operator, where $C(L)$ can be expanded as $C(L)=C(1)+C^{*}(L)(1-L)$, see e.g. Engle \& Granger (1987). Accordingly, the representation of $Y_{t}$ is given by,

$$
Y_{t}=\frac{C(1)}{1-L} \varepsilon_{t}+C^{*}(L) \varepsilon_{t}=C(1) \sum_{i=1}^{t} \varepsilon_{t-i}+C^{*}(L) \varepsilon_{t}
$$

where $C(1)$ can be found from the cointegration parameters and will have reduced rank $p-r=n$, i.e. only $n$ elements of $C(1) \sum \varepsilon_{t-i}$ have independent permanent effect on 
$Y_{t}$. The cointegrated $V A R$ model can thus be represented as common stochastic trends plus stationary disturbances.

In order to illustrate the concepts, let $Y_{t}$ contain five integrated and non-stationary time series, $p=5$. If we cannot reject four cointegration vectors $(r=4)$, the rank of $C$ (1) cannot be rejected to equal one $(n=p-r)$, i.e. the non-stationary behavior of the five individual time series in $Y_{t}$ would be generated by one underlying common stochastic trend.

\subsection{Estimation of the cointegration model - recursive and rolling tests}

When testing for cointegration and the number of common trends, Johansen (1988, 1991) shows that the solution to the eigenvalue problem $\left|\lambda S_{11}-S_{10} S_{00}^{-1} S_{01}\right|=0$, where $S_{i j}=T^{-1} \sum_{t=1}^{T} R_{i t} R_{j t}^{\prime}$, for $i, j=0,1$ and $R_{0 t}, R_{1 t}$ are the residuals from the regressions of $\Delta Y_{t}$, respectively $Y_{t}$, on $\Delta Y_{t-1}, \ldots, \Delta Y_{t-k-1}$, will give $p$ eigenvalues $1 \geq \hat{\lambda}_{1} \geq \ldots \geq$ $\widehat{\lambda}_{p} \geq 0$ and $\widehat{\beta}=\left(\widehat{v}_{1}, \ldots, \widehat{v}_{p}\right)$ where $\widehat{v}_{1}, \ldots, \widehat{v}_{p}$ are the associated normalized eigenvectors. The maximized value of the likelihood function is given by $\mathcal{L}^{-\frac{2}{T}}=\left|S_{\mathbf{0 0}}\right| \Pi_{i=1}^{p}\left(1-\hat{\lambda}_{i}\right)$. In order to determine the number of statistically significant cointegration vectors (the number of $\hat{\lambda}$ s being statistically different from zero), Johansen $(1988,1991)$ shows that a likelihood ratio test statistic for $r$ cointegration vectors in a space with a maximum of $p$ cointegration vectors is given by:

$$
-2 \ln Q(r \mid p)=-T \sum_{i=r+1}^{p} \ln \left(1-\hat{\lambda}_{i}\right)
$$

The asymptotic distribution of the likelihood ratio test statistic in (3) is derived in Johansen \& Juselius (1990) who also present critical values, as do Osterwald-Lenum (1992).

When actually determining the number of cointegration vectors/common stochastic trends, a sequential testing strategy is used. First, the hypothesis of $r=0$ is tested against the alternative of $r=p$ (i.e. all series being unit root series against the alternative hypothesis of all series being stationary series); $\mathcal{H}(r=0 \mid r=p)$. If this test is rejected (and thus $\lambda_{1}>0$ ), the hypothesis of at most one cointegration vector, $r \leq 1$, is tested against the alternative hypothesis of $r=p ; \mathcal{H}(r \leq 1 \mid r=p)$, and so forth until the hypothesis of $r \leq p-1$ is tested against $r=p ; \mathcal{H}(r \leq p-1 \mid r=p)$. When a particular hypothesis cannot be rejected, the sequential testing procedure stops. 
Recursive tests. Generally, when estimating a model recursively the initial observation is kept fixed and the sample length is increased by adding an additional observation at each recursive estimation. Hansen \& Johansen $(1992,1998)$ show that in the recursive cointegration analysis the $p-r$ smallest eigenvalues will converge towards zero, while the $r$ largest eigenvalues converge towards the solution to $\left|\lambda \beta^{\prime} \Sigma_{11} \beta-\beta^{\prime} \Sigma_{10} \Sigma_{00}^{-1} \Sigma_{01} \beta\right|=0$, where $\beta^{\prime} \Sigma_{11} \beta$ and $\Sigma_{\mathbf{0} 0}$ are the asymptotic variances of $\beta^{\prime} R_{1 t}$ and $R_{0 t}$, respectively, and $\beta^{\prime} \Sigma_{10}$ is the asymptotic covariance matrix for $\beta^{\prime} R_{1 t}$ and $R_{0 t}$ (see e.g. Theorem 1 , Hansen \& Johansen, 1998). Therefore, based on recursive estimations of the $V A R$-model, a sequence of likelihood ratio test statistics as given by (3), should be increasing for tests of the hypotheses that the truly positive cointegration vectors equal zero and should be constant for tests of the hypotheses that the truly non-significant cointegration vectors equal zero.

Rolling tests. Generally, when computing rolling test statistics, the size of the subsample is kept constant and both the first and the last observation in the subsample roll through the full sample. The size of the subsample is thus a constant fraction of the size of the full sample.

2.2.1 Discussion of the recursive and the rolling tests. As mentioned, in the recursive estimations, the path of the likelihood ratio test statistics should be increasing for tests of the hypotheses of "truly" positive eigenvalues being equal to zero due to the inclusion of additional observations to the sample. Based on some constant critical value for the particular hypothesis being tested, an increasing test statistic implies that the probability of rejecting a "false" hypothesis increases. In our recursive estimations, an increasing path of the test statistics could thus be due to both $(i)$ an actual increase in the number of cointegration vectors or $(i i)$ adjustment towards the asymptotic properties of the particular tests. On the other hand, the rolling tests makes it possible to evaluate whether a finding of increasing test statistics in the recursive tests is due to actual convergence and not the inclusion of additional observations in itself. In particular, increasing test statistics for the rolling regressions will indicate an increase in the stance of actual economic convergence (an increasing number of cointegration vectors cannot be rejected), as the number of observations in each subsample is kept constant. ${ }^{5}$

\footnotetext{
${ }^{5} \mathrm{On}$ the other hand, the advantage of the recursive regressions is inter alia that all historic information is taken into account. Furthermore, for a non-increasing sequence of test statistics in a recursive estimation, the hypothesis of increased convergence is obviously rejected.
} 


\subsection{Interpretation of the number of common stochastic trends}

In the statistical framework we use, one can think of two interpretations of a declining number of common stochastic trends: increasing stationarity of the relevant time series or the relevant time series being increasingly driven by the same shocks with permanent effects. In this section, we discuss these two interpretations in terms of possible ERM exchange rate convergence.

Stationarity of the time series. In a statistical sense, the de facto presence of the ERM target zone bands implied that the first two moments of the exchange rate processes were bounded, if the target zone bands could not be changed, i.e. nonstationary exchange rate processes could implicitly be ruled out when the target zones remained constant (in addition, the Maastricht Treaty stipulated that the target zone bands were not allowed to be changed during the last two years before introducing the euro). Furthermore, any stationary time series in a cointegration framework is represented by a specific cointegration vector, such as e.g. $\beta^{\prime}=(0,0,1,0,0)$. On the other hand, because the target zone bands actually did change during the ERM period, also ERM exchange rates can be modelled as non-stationary processes. It follows that if convergence of ERM exchange rates is followed by fewer changes in the target zone bands and thus more stationary exchange rate processes, an increasing number of stationary exchange rate series (implying an increasing number of cointegration vectors) should be found - leading to a decrease in the number of common stochastic trends characterizing the system of exchange rates. When the process of converging has come to an end and no more changes in the target zones occur, one would then expect all exchange rate series to be stationary, i.e. finding $p$ cointegration vectors and zero common trends.

Generally, we would interpret a finding of zero common stochastic trends at sample end as a "perfectly" converged system.

Shock structure. Since the work of Nelson \& Plosser (1982) it has been recognized that an univariate difference-stationary time series can be decomposed into a permanent component plus a transitory disturbance. For a multivariate system of non-stationary time series the same holds, i.e. the non-stationary system is driven by permanent components plus transitory disturbances. If cointegration is established, the number of components with permanent effect is reduced and (some of) the time series in the system are driven by linear combinations of the reduced number of shocks with permanent 
effects, see e.g. Stock \& Watson (1988), King et al. (1991), and the representation in (2). For this reason, if all time series actually remain non-stationary during the period where the number of common stochastic trends declines, the non-stationarity will be caused by fewer shocks with a permanent effect.

Therefore, even if all individual exchange rates towards the German mark remain non-stationary, finding a decreasing number of common stochastic trends should still be interpreted as a sign of convergence, as the exchange rates would follow increasingly similar time-series processes. In particular, if there is only one shock with a permanent effect on the $p$ non-stationary time series, one should expect to find $p-1$ cointegration vectors.

Generally, we would not interpret a finding of one (or more) common stochastic trends at sample end as a "perfectly" converged system because shocks to the individual time series would still have permanent effect and the first two moments of the time series would thus not be bounded.

\subsection{Convergence criteria and exchange rate quotation}

At this stage of the analysis, it seems relevant to comment on the apparent difference between our condition for "perfect convergence" (of exchange rates) and the condition for "perfect convergence" used by Bernard \& Durlauf (1995) who investigate convergence of real production series. Where we define perfect convergence as a situation where our $V A R$-system of exchange rates is driven by zero common stochastic trends, Bernard \& Durlauf (1995) define perfect convergence as a situation where the VAR-system they analyze is driven by a single common stochastic trend. Basically, the reason for this apparent difference is that when considering exchange rates quoted vis á vis another currency within the $V A R$-system (as for instance in this paper where we quote exchange rates as the prices of one German mark in terms of the other ERM currencies), the obvious requirement for convergence is that all exchange rates are stationary. On the other hand, when considering time series such as real activity, one would naturally allow those individual series to be non-stationary time series, also when converged, with the requirement for convergence then being that all the series are driven by the same shock with a permanent effect.

Analogously, if we had used exchange rates quoted against a currency that does not belong to the ERM target zone system, e.g. the US dollar, and all the exchange rates in this alternative $V A R$-system were non-stationary, it would have been relevant to use 
the Bernard \& Durlauf (1995) requirement for "perfect convergence", as the necessary condition for ERM exchange rate convergence would then be that the intra-ERM crossrates were stationary. ${ }^{6}$

A simple example illustrates these points. Consider the two exchange rates $e_{1}=$ $\ln (F R F / U S D)$ and $e_{2}=\ln (D E M / U S D)$, these being the logarithms to the prices in French franc respectively German mark for one US dollar. In this case, the necessary condition for intra-ERM convergence imposes a particular restriction on the cointegration vector, this being that $\ln (F R F / U S D)-\ln (D E M / U S D)=\ln (F R F / D E M)$ is stationary, i.e. the logarithm to the price of one German mark in French franc should be stationary. For a $V A R$-system including the two exchange rates $e_{1}$ and $e_{2}$ (i.e. $p=2$ ), one cointegration vector (i.e. $r=1$ ), as $\beta^{\prime}=[1,-1]$, and thus one common stochastic trend should be expected (i.e. $n=p-r=1$ ).

Consequently, when the exchange rates are quoted as the prices of one German mark in terms of other ERM currencies, as in our case, these series should be stationary by themselves; for the model with one exchange rate quoted in German mark (i.e. $p=1$ ), one thus expects one cointegration vector (i.e. $r=1$ ), as $\beta^{\prime}=1$, and thus zero common stochastic trends (i.e. $n=p-r=0$ ).

In conclusion, when measuring convergence of exchange rates within the ERM with exchange rates quoted as prices for one German mark in terms of the other ERM currencies (as we do), the necessary condition for a convergence process having already come to an end, is that all exchange rates should be stationary and there should be zero common stochastic trends.

\section{Data description and filtering procedures}

The data which we analyze in the following sections consists of weekly observations (Wednesdays, or Thursday if Wednesday is not available) on exchange rates and onemonth Euro market interest rate spreads. Data was collected for the countries that have belonged to the ERM since March 13, 1979; the Irish pound is, however, omitted in our empirical analysis due to data limitations. The exchange rate data consists of exchange rates for the Belgian franc, the Danish kroner, the French franc, the Italian lira, and the Dutch guilder all versus the German mark. The relevant one-month Euro market interest rate spreads are based on bid rates supplied by the Bank of International

\footnotetext{
${ }^{6}$ The single common stochastic trend would then represent the trend of all the ERM currencies versus the US dollar.
} 
Settlements. The final decision regarding the number of currencies to be replaced by the euro, was made at the EU summit on May 1-3, 1998 and based on the convergence report as released on March 25, 1998. Therefore, we let our sample period run from March 13, 1979 (where the ERM was initiated) until April 1, 1998 and each data series includes a total of 995 observations. In addition to the analysis of the actually observed exchange rates, we also analyze shadow exchange rates. Based on the interest rate spreads and the exchange rates, the shadow exchange rates are extracted through two filters: a non-linear filter and a linear filter. ${ }^{7}$

\subsection{A non-linear filter for extracting the shadow exchange rates}

The particular non-linear filter that we use is taken from Rangvid \& Sørensen (2000), and in this section we will briefly review their model. ${ }^{8}$ Basically, Rangvid \& Sørensen (2000) present a general theoretical framework for the dynamics of an exchange rate where the fluctuations of the exchange rate are restricted by a non-credible target zone regime (i.e. a target zone that can be realigned). ${ }^{9}$

The basic feature of the target zone modelling is thus that the exchange rate is restricted to move within a band unless a realignment occurs. The timing of realignments is described by a so-called Cox process which is basically a Poisson process with stochastic intensity. In particular, the intensity depends on the position of the exchange rate within the target zone band as well as how misaligned the exchange rate is relatively to the shadow exchange rate.

Formally, the dynamics of the (log-) shadow exchange rate $f_{t}$ and the (log-) exchange

\footnotetext{
${ }^{7}$ We do not claim that these two filtering procedures necessarily span the whole set of possible filtering procedures. What we do claim, though, is simply that the two procedures represent two distinctly different ways to extract shadow exchange rates: a non-linear and a linear procedure. For a more detailed review of the target zone literature and the currency crises literature, both of which pay careful attention to the role played by the shadow exchange rate, see Garber \& Svensson (1995). Furthermore, Flood et al. (1991) and Bartolini \& Prati (1997) present and discuss other simple linear filters through which measures of shadow exchange rates can be obtained.

${ }^{8} \mathrm{~A}$ more detailed discussion of the particular specification of the model, the estimation procedure, as well as the parameter estimates can thus be found in Rangvid \& Sørensen (2000).

${ }^{9}$ The model is a variation of the model in Christensen et al. (1998). Our motivation for extending the particular model of Christensen et al. (1998) is due to some of the desirable features of that model. For instance, the model allows for a positive correlation between the exchange rate and the interest rate during periods with pressure on the exchange rate; a feature which is in accordance with the results from empirical tests, as noted by Bertola \& Caballero (1992), but not present in for instance the basic Krugman (1991) model.
} 
rate $e_{t}$ are described by the following stochastic differential equation system,

$$
\begin{aligned}
& d f_{t}=\mu d t+\sigma d W_{1 t} \\
& d e_{t}=m_{t} d t+v_{t} d W_{2 t}+\epsilon_{t} d N_{t}
\end{aligned}
$$

where $W_{1 t}$ and $W_{2 t}$ are wiener-processes with $\operatorname{cov}\left(d W_{1 t}, d W_{2 t}\right)=\rho d t, N_{t}$ is a Cox process with jump intensity $\lambda_{t}$, and $\epsilon_{t}$ is the jump size if a realignment occurs at time $t$. The drift and volatility of the shadow exchange rate, $\mu$ and $\sigma$, are constant parameters whereas the drift and volatility of the exchange rate are given by,

$$
m_{t}= \begin{cases}a\left(\theta_{t}-e_{t}\right)+\kappa\left(\frac{u_{t}-e_{t}}{u_{t}-\theta_{t}}\right)\left(f_{t}-e_{t}\right) & \text { if } \theta_{t}<e_{t}<f_{t} \\ a\left(\theta_{t}-e_{t}\right)+\kappa\left(\frac{e_{t}-l_{t}}{\theta_{t}-l_{t}}\right)\left(f_{t}-e_{t}\right) & \text { if } f_{t}<e_{t}<\theta_{t} \\ a\left(\theta_{t}-e_{t}\right)+\kappa\left(f_{t}-e_{t}\right) & \text { otherwise }\end{cases}
$$

and,

$$
v_{t}=\delta \sqrt{\frac{4\left(u_{t}-e_{t}\right)\left(e_{t}-l_{t}\right)}{\left(u_{t}-l_{t}\right)^{2}}}
$$

where $a, \kappa$, and $\delta$ are constant parameters and $\theta_{t}, l_{t}$, and $u_{t}$ denote the $(\log$-) central parity, the (log-) lower bound of the band, and the (log-) upper bound of the band, respectively.

The parameter $a$ describes the degree of tendency towards the central parity and $\kappa$ describes the degree of tendency towards the shadow exchange rate. The tendency towards $f_{t}$ evaporates at the boundaries whenever this tendency would otherwise pull it outside the exchange rate band. Likewise, the volatility $v_{t}$ evaporates at the boundaries in order to ensure that the target zone is sustained unless a realignment jump occurs. The parameter $\delta$ can be interpreted as the volatility of the exchange rate in the center of the target zone.

The realignment intensity and the exchange rate jump sizes at a realignment date are described by,

$$
\lambda_{t}=\lambda_{0}+\lambda_{1} \max \left[0,\left(f_{t}-e_{t}\right)\left(\frac{e_{t}-\theta_{t}}{u_{t}-l_{t}}\right)\right] \quad \text { and } \quad \epsilon_{t}=\left(f_{t}-e_{t}\right)+\eta_{t}
$$

respectively, where $\lambda_{\mathbf{0}}$ and $\lambda_{1}$ are non-negative constant parameters and $\eta_{t}$ is a series of independent and normally distributed stochastic variables with mean zero and standard deviation $\omega$. Hence, the realignment intensity depends on the distance to the central parity of the target zone as well as the distance to the shadow exchange rate and, if there is a realignment, the exchange rate will jump to a position around the shadow exchange rate. 
Imposing uncovered interest rate parity, the interest rate spread must equal the drift rate in the (log-) exchange rate plus a component representing the rate of expected $\left(\log _{-}\right)$ exchange rate movement due to a potential realignment:

$$
i_{t}-i_{t}^{f}=m_{t}+\lambda_{t}\left(f_{t}-e_{t}\right)
$$

The interest rate spread in (8) is a monotonically increasing function of $f_{t}$ and there is thus an invertible relationship between the interest rate spread and the shadow exchange rate.

When referring to "shadow exchange rates extracted through a non-linear filter" in what follows, we thus refer to $f_{t}$ as filtered out through the inverted relationship in (8) for given observed interest rate spreads. When filtering out the shadow exchange rates, we use the approximate maximum likelihood parameter estimates reported in Rangvid \& Sørensen (2000). ${ }^{10}$ Especially, the parameters of the target zone model are estimated separately for each currency (in relation to the German mark), i.e. the non-linear filter allows for different estimated parameters across the different exchange rates.

In order to evaluate our findings with respect to the restrictions embodied in the use of a particular non-linear model for the ERM exchange rates, we also investigated the actually observed exchange rates as well as shadow exchange rates extracted through a more simple linear filter. In the linear filter, we imposed equal parameters across the different exchange rates, so as not to rely on any particular estimation procedure.

\subsection{A linear filter for extracting the shadow exchange rates}

To obtain the shadow exchange rates through a linear filter we rely on the recent results from "fundamental-based" approaches to exchange rates, as reported in for instance Chinn \& Meese (1995), Mark (1995), Mark \& Choi (1997), and MacDonald (1999). These authors show that if exchange rates are assumed to follow processes such as,

$$
e_{t+1}-e_{t}=-\gamma\left(e_{t}-f_{t}\right)
$$

where $e_{t}$ is the logarithm to the exchange rate quoted as the price of foreign currency measured in domestic currency and $f_{t}$ is the logarithm to the shadow exchange rate, the shadow exchange rate has predicting power for the future long-horizon development of the exchange rate. Imposing uncovered interest parity, in the sense that the spread

\footnotetext{
${ }^{10}$ The estimation approach in Rangvid \& Sørensen (2000) is somewhat related to the estimation approach in Bekaert \& Gray (1998).
} 
between the return on a domestic bond, $i$, and the return on a foreign bond, $i_{t}^{f}$, equals the expected future change in the exchange rate, i.e. $i_{t}-i_{t}^{f}=\left(e_{t+1}-e_{t}\right)$, we can back out the shadow exchange rate as:

$$
f_{t}=e_{t}+\gamma^{-1}\left(i_{t}-i_{t}^{f}\right)
$$

In order to filter out the shadow exchange rate, we need an estimate of $\gamma$. Mark (1995) estimates the range for $\gamma$ to be between zero and 1.3. When referring to "shadow exchange rates extracted through a linear filter" in what follows, we thus refer to $f_{t}$ as filtered out through the solution in (9) with $\gamma=1$ imposed on all currencies.

Finally, as mentioned, we also investigated the dynamics of convergence for the values of the exchange rates that we actually observe. In the following, we refer to these as the "actually observed exchange rates". ${ }^{11}$

\section{Convergence in the ERM}

We now turn to the empirical analysis of convergence within the ERM, i.e. we investigate whether the number of common stochastic trends in the systems of ERM (shadow) exchange rates has decreased during the ERM period. We initially note that finding a reduced but positive (i.e. smaller than $p$ but larger than zero) number of cointegration vectors implies that some (and possibly all) of the time series contained in $Y_{t}$ are integrated and non-stationary, and that the necessary condition for "perfect" convergence (zero common trends) is thus rejected.

\subsection{Recursive tests for the number of cointegration vectors/common trends}

The recursive test statistics for the number of cointegration vectors and common stochastic trends, based on (3) and scaled by their 90 percentage asymptotic critical confidence values (from Osterwald-Lenum, 1992), are plotted in Figure 1, with the period 1979 to 1980 reserved for initial estimates.

\section{[INSERT FIGURE 1 ABOUT HERE]}

\footnotetext{
${ }^{11}$ One can think of the "actually observed exchange rates" as exchange rates extracted through the linear filter (9) with $\gamma^{-1}=0$ imposed on all exchange rates. Furthermore, by using two linear filters, we try to make a perspective on the sensitivity of the results with respect to the choice of a particular value for $\gamma$.
} 
Figure 1 plots the scaled recursive tests from three five-dimensional VARs of order four (i.e. with four lags) where the constant in each $V A R$ is left unrestricted. ${ }^{12}$ The VARs include, respectively, the actually observed ERM exchange rates, the ERM shadow exchange rates extracted through the non-linear filter as described in section 3.1, and the ERM shadow exchange rates extracted through a linear filter as described in section 3.2 .

Common to all filtering procedures is the basic overall tendency for the tests not to reject an increasing number of cointegration vectors and thus a decreasing number of common stochastic trends since 1983, as the test statistics for the hypotheses $\mathcal{H}_{0}^{i}: r \leq i$, for $i=0, \ldots, 3$ (4 since 1996) are generally upward sloping.

Therefore, the findings indicate that in the early years of the ERM - until 1983 the hypotheses of $r=0$ cannot be rejected, implying that all exchange rates were nonstationary and described by their own stochastic trend during this early subsample. After 1983, the test statistics start to increase, and a process of convergence begins. From the late 1980s and onwards two cointegration vectors cannot be rejected and the results for the recent years point towards a third cointegration vector not being rejected. Finally, an important exception to the general tendency of decreasing numbers of common stochastic trends is seen during the 1992-1993 period.

It is furthermore noted that at the end of the sample period, the hypothesis of "perfect" convergence is clearly rejected regardless of the specific filter used. This implies that even when we find that the (shadow) exchange rates have been within a process of converging since 1983, the (shadow) exchange rates had not converged "perfectly" as the decision with respect to the number of currencies to be replaced with the euro was finally taken - when evaluated over the full sample.

Finally, even though the general tendency of a decrease in the number of common stochastic trends does not seem to depend on the specific filtering procedure, the graphical profiles of the test statistics do, to a minor extent, depend of the specific measure of $f_{t}$ included in the VARs. In particular, the linear filter indicates the relatively highest degree of convergence at the end of the sample period. As it is also the linear filter which puts the highest weight on the interest rate spread, this finding probably reflects a convergence in interest rate spreads in addition to the convergence in nominal exchange rates.

\footnotetext{
${ }^{12}$ We experimented with different choices of $k$. Basically, the results were rather robust with respect to the choice of lags to include in the VAR.
} 


\subsection{Rolling tests for the number of cointegration vectors}

In addition to the full-sample recursive tests presented above, we also looked upon shorter-sample tests of the convergence hypothesis by computing rolling regressions where the number of observations is kept constant. We computed the rolling regressions with a constant sample size of 156 observations (3 years). ${ }^{13}$ The paths of scaled likelihood ratio test statistics are shown in Figure 2. For ease of interpretation, the figure shows the paths of the tests statistics for all three filters for $\mathcal{H}(r=0 \mid r=p)$ in the upper-left graph, the second graph in the first column shows the sample paths of the tests statistics for $\mathcal{H}(r \leq 1 \mid r=p)$ for all three filters, and so forth.

\section{[INSERT FIGURE 2 ABOUT HERE]}

At the overall level, the rolling tests confirm the insights from the recursive tests of no convergence during the 1979-1983 period and, on the other hand, increasingly converging ERM exchange rates during the 1983-1998 period, with a possible minor interruption during the ERM turmoils of 1992-1993.

Especially, Figure 2 confirms that all exchange rates were described by their own stochastic trend during the 1979-1983 period (in Figure 2, the series are ordered such that the test statistic for a particular subsample is placed at the end of the rolling sample). On the other hand, where the recursive tests indicated a rather stable process of convergence during 1983-1998, the rolling tests indicate some larger interruptions of this process in 1986 and 1992-1993. What thus appear to be only minor interruptions of the basic convergence process when evaluated over the whole period after 1983, as in the recursive tests, appear more significant when evaluated over their own shorter sample in isolation. Finally, and important to point out, when based on a three-year window, the rolling tests indicate that the ERM exchange rates were "perfectly converged" in 1998, in the sense that all exchange rates are found to be stationary when evaluated over the 1995-1998 period. This contrasts to the finding of two (or three) common stochastic trends at sample end in Figure 1.

When assessing the evidence based on both methods of time-varying tests, a tentative conclusion with respect to the degree of convergence in the different subperiods could be lined up as in Figure 3 .

\footnotetext{
${ }^{13}$ In the working paper version of the paper, also ten-year rolling windows (i.e. a sub-sample size of 520 observations) were analyzed. Basically, these tests supported the qualitative results presented in this section. The tests are available upon request.
} 


\section{[INSERT FIGURE 3 ABOUT HERE]}

Comparing with the history of ERM exchange rates (see e.g. Buiter et al., 1998 and Gros \& Thygesen, 1998), these findings are intuitively interpretable. Until 1983, realignments within the ERM were not unusual events, as the exchange rates were used to influence the business cycles of the individual ERM countries, i.e. the exchange rate policies were not especially focused on avoiding realignments. From 1987 to 1992 only one realignment was realized (associated with the narrowing of the fluctuation band around the Italian lira central parity in 1990). In 1992-1993, the ERM was exposed to the worst pressure in its history resulting in the Italian lira leaving the ERM and the fluctuation band being widened to \pm 15 percentage for the Belgian franc, the Danish kroner, and the French franc, while maintained at \pm 2.25 percentage for the Dutch guilder versus the German mark. Finally, in the most recent years some emphasis has been directed towards preparing for the introduction of the common currency and thus on coordinating the exchange rate policies, even with wide bands.

\subsection{Declining numbers of common stochastic trends: stationarity or change in the structure of shocks?}

In section 2.3, we discussed two interpretations of the relationship between convergence and declining numbers of common stochastic trends: $(i)$ stationarity of the relevant time series or $(i i)$ the relevant non-stationary time series being increasingly driven by the same shocks. In this section, we will test whether explanation $(i)$ can explain our findings.

As the cointegration procedure reviewed in section 2 is a multivariate extension of the basic univariate Dickey-Fuller procedure, one way to proceed is to conduct simple recursive Dickey-Fuller tests in order to test whether each individual time series becomes increasingly stationary, as the sample period is extended. ${ }^{14}$ Figure 4 presents the results from recursive Dickey-Fuller tests of each single (shadow) exchange rate. The test statistics (based on Dickey-Fuller regressions with four augmentation lags and a constant, but no trend) are scaled by their critical 90 percentage confidence values and,

\footnotetext{
${ }^{14}$ We also conducted rolling Dickey-Fuller tests. Basically, these tests supported the division into phases of convergence as illustrated in Figure 3, and can be obtained upon request. Furthermore, an early analysis of the unit-root properties of nominal floating exchange rates is given in Meese \& Singleton (1982) while Anthony \& MacDonald (1998) investigate the unit root properties of single ERM exchange rate series.
} 
hence, a scaled test statistic below one, at time $t$, suggests that the specific (shadow) exchange rate cannot be rejected to contain an unit root over the period from March 13, 1979 to time $t$. The time series in the graphs thus present such tests for $t$ increasing and the first observation remaining at March 13, 1979.

On the basis of the path of scaled recursive Dickey-Fuller test statistics, we are led to believe that all series, possibly with the exception of the Italian lira series, seem to behave more and more as stationary time series as the sample period is extended.

\section{[INSERT FIGURE 4 ABOUT HERE]}

The graphs in Figure 4 add some additional insights to the interpretation of Figure 1. Especially, at sample end, Figure 1 suggests two or three common stochastic trends; the specific number of common stochastic trends being dependent upon the specific measure of $f_{t}$ used in the VAR. From the recursive Dickey-Fuller tests, it appears that only the Danish kroner and the Dutch guilder exchange rates are definitely stationary and only the Italian lira series seems to be definitely non-stationary, whereas the Belgian franc and the French franc are in-between cases, i.e. the number of common stochastic trends suggested in Figure 1 seems to be in accordance with the findings from the recursive Dickey-Fuller tests. Finally, the tendency for an increase in the degree of convergence appears only after 1983 also for the univariate Dickey-Fuller tests.

\section{Conclusion}

To sum up; in this paper we proposed to use time-varying multivariate cointegration techniques when studying the dynamics of convergence of several exchange rates. In the illustration of the procedures, we found that ERM exchange rates tended to behave in an increasingly similar manner as January 1, 1999 approached.

In addition to the analysis presented here, the hypothesis of a reduced number of common stochastic trends for currencies joining a monetary union is general and can be applied to possible future applicants (the "pre-ins") wanting to introduce the euro as national currency (e.g. East European countries). Furthermore, for countries pegging their exchange rate towards another country (even if these countries do not intend to join a currency union), one could expect a declining number of common stochastic trends, if the credibility of the peg is believed to be increased over time. More generally, the procedures can be used to test for the dynamics of convergence for other types of economic variables, such as real GDP, stock prices (see Rangvid, 2000), and so forth. 


\section{References}

Anthony, Myrvin, and Ronald MacDonald (1998): "On the Mean-reverting Properties of Target Zone Exchange Rates: Some Evidence from the ERM," European Economic Review, 42(8), 1493-1523.

Baillie, Richard T., and Tim Bollerslev (1989): "Common Stochastic Trends in a System of Exchange Rates," Journal of Finance, 44(1), 167-181.

— (1994): "Cointegration, Fractional Cointegration, and Exchange Rate Dynamics," Journal of Finance, 49(2), 737-745.

Bartolini, Leonardo, and Alessandro Prati (1997): "Soft versus Hard Targets for Exchange Rate Intervention," Economic Policy, 24, 13-52.

Bekaert, Geert, and Stephen F. Gray (1998): "Target Zones and Exchange Rates: An empirical investigation," Journal of International Economics, 45(1), 1-35.

Bernard, Andrew B., and Steven N. Durlauf (1995): "Convergence in International Output," Journal of Applied Econometrics, 10(2), 97-108.

- (1996): "Interpreting Tests of the Convergence Hypothesis," Journal of Econometrics, $71(1-2), 161-173$.

Bertola, Giuseppe, and Ricardo J. Caballero (1992): “Target Zones and Realignments," American Economic Review, 82(3), 520-536.

Buiter, William H., Giancarlo Corsetti, and Paolo A. Pesenti (1998): Financial Markets and European Monetary Coorperation - the lessons of the 1992-1993 Exchange Rate Mechanism crisis. Cambridge University Press.

Chinn, Menzie D., and Richard A. Meese (1995): "Banking on Currency Forecasts: How Predictable is the Change in Money?," Journal of International Economics, 38(1/2), $161-178$.

Christensen, Peter O., David Lando, and Kristian Miltersen (1998): "State-dependent Realignments in Target Zone Currency Regimes," Review of Derivatives Research, 1(4), $295-323$.

Diebold, Francis X., Javier Gardeazalbal, and Kamil Yilmaz (1994): "On Cointegration and Exchange Rate Dynamics," Journal of Finance, 49(2), 727-735.

Durlauf, Steven N., and Danny T. Quah (1999): “The New Empirics of Economic Growth," in Handbook of Macroeconomics, ed. by John B. Taylor, and Michael Woodford, vol. 1A, Part 1, chap. 4, pp. 231-304. Elsevier Science Publishers B.V. 
Eichengreen, Barry (1997): European Monetary Integration. The MIT Press.

Engle, Robert F., and Clive W. J. Granger (1987): "Co-Integration and Error Correction: Representation, Estimation and Testing," Econometrica, 55(2), 251-276.

Flood, Robert P., Andrew K. Rose, and Donald J. Mathieson (1991): "An Empirical Exploration of Exchange-Rate Target-Zones," Carnegie-Rochester Conference Series on Public Policy, 35, 7-77.

Garber, Peter M., and Lars E. O. Svensson (1995): "The Operation and Collapse of Fixed Exchange Rate Regimes," in Handbook of International Economics, ed. by G. Grossman, and K. Rogoff, vol. III, chap. 36, pp. 1865-1911. Elsevier Science Publishers B.V.

Gros, Daniel, and Niels Thygesen (1998): European Monetary Integration: From the European Monetary System to Economic and Monetary Union. Longman.

Hall, Stephen G., Donald Robertson, and Michael R. Wickens (1992): "Measuring Convergence of the EC Economies," The Manchester School, LX(Supplement), 99-111.

(1997): "Measuring Economic Convergence," International Journal of Finance and Economics, 2(1), 131-143.

Hansen, Henrik, and Søren Johansen (1992): "Recursive Estimation in Cointegrated VARModels," Discussion Paper, Institute of Economics, University of Copenhagen, 92-13.

- (1998): "Some Tests for Parameter Constancy in Cointegrated VAR-Models," mimeo.

Johansen, Søren (1988): "The Statistical Analysis of Cointegration Vectors," Journal of Economic Dynamics and Control, 12(2), 231-254.

(1991): "Estimation and Hypothesis Testing of Cointegration Vectors in Gaussian Vector Autoregressive Models," Econometrica, 59(6), 1551-1580.

- (1995): Likelihood Based Inference in Cointegrated Vector Autoregressive Models. Advanced Texts in Econometrics. Oxford University Press.

Johansen, Søren, and Katarina Juselius (1990): "Maximum Likelihood Estimation and Inference on Cointegration - With Applications to the Demand for Money," Oxford Bulletin of Economics and Statistics, 52(2), 169-210.

Kenen, Peter B. (1995): Economic and Monetary Union in Europe. Cambridge University Press.

King, Robert G., Charles I. Plosser, James H. Stock, and Mark W. Watson (1991): "Stochastic Trends and Economic Fluctuations," American Economic Review, 81(4), 819-840. 
Krugman, Paul R. (1991): "Target Zones and Exchange Rate Dynamics," Quarterly Journal of Economics, 106(3), 669-682.

MacDonald, Ronald (1999): "Exchange Rate Behaviour: are Fundamentals Important?," Economic Journal, 109, 673-691.

Mark, Nelson C. (1995): "Exchange Rates and Fundamentals: Evidence on Long-Horizon Predictability," American Economic Review, 85(1), 201-218.

Mark, Nelson C., and Doo-Yull Choi (1997): "Real Exchange-Rate Prediction over Longer Horizons," Journal of International Economics, 43(1/2), 29-60.

Meese, Richard A., and Kenneth J. Singleton (1982): "On Unit Roots and the Empirical Modelling of Exchange Rates," Journal of Finance, 37(4), 1029-1035.

Nelson, Charles R., and Charles I. Plosser (1982): "Trends and Random Walks in Macroeconomic Time Series," Journal of Monetary Economics, 10(2), 139-162.

Osterwald-Lenum, Michael (1992): "A Note with Quantiles of the Asymptotic Distribution of the Maximum Likelihood Cointegration Rank Statistic: Four Cases," Oxford Bulletin of Economics and Statistics, 54(3), 461-471.

Perron, Pierre (1989): "The Great Crash, the Oil Price Shock, and the Unit Root Hypothesis," Econometrica, 57(6), 1361-1401.

Rangvid, Jesper (2000): "Increasing Convergence among European Stock Markets? - a recursive common stochastic trends analysis." Forthcoming in Economics Letters.

Rangvid, Jesper, and Carsten Sørensen (2000): "Determinants of the Implied Shadow Exchange Rates from a Target Zone." Forthcoming in European Economic Review.

Stock, James H., and Mark W. Watson (1988): “Testing for Common Trends," Journal of American Statistical Association, 83, 1097-1107. 
Figure 1: Recursive multivariate tests for the number of common trends

The figures show recursive tests for the number of cointegration vectors, scaled by their critical 90 percentage confidence values, in $V A R$ models containing respectively five ERM exchange rates, five shadow exchange rates from linear filters, and five shadow exchange rates from nonlinear filters. The period March 13, 1979 to January 1, 1980 is reserved for initial estimates. A series above "one" suggests rejection of the hypothesis of respectively $r \leq i, i=0,1,2,3,4$ and, hence, the number of series below "one" indicates the number of common stochastic trends.
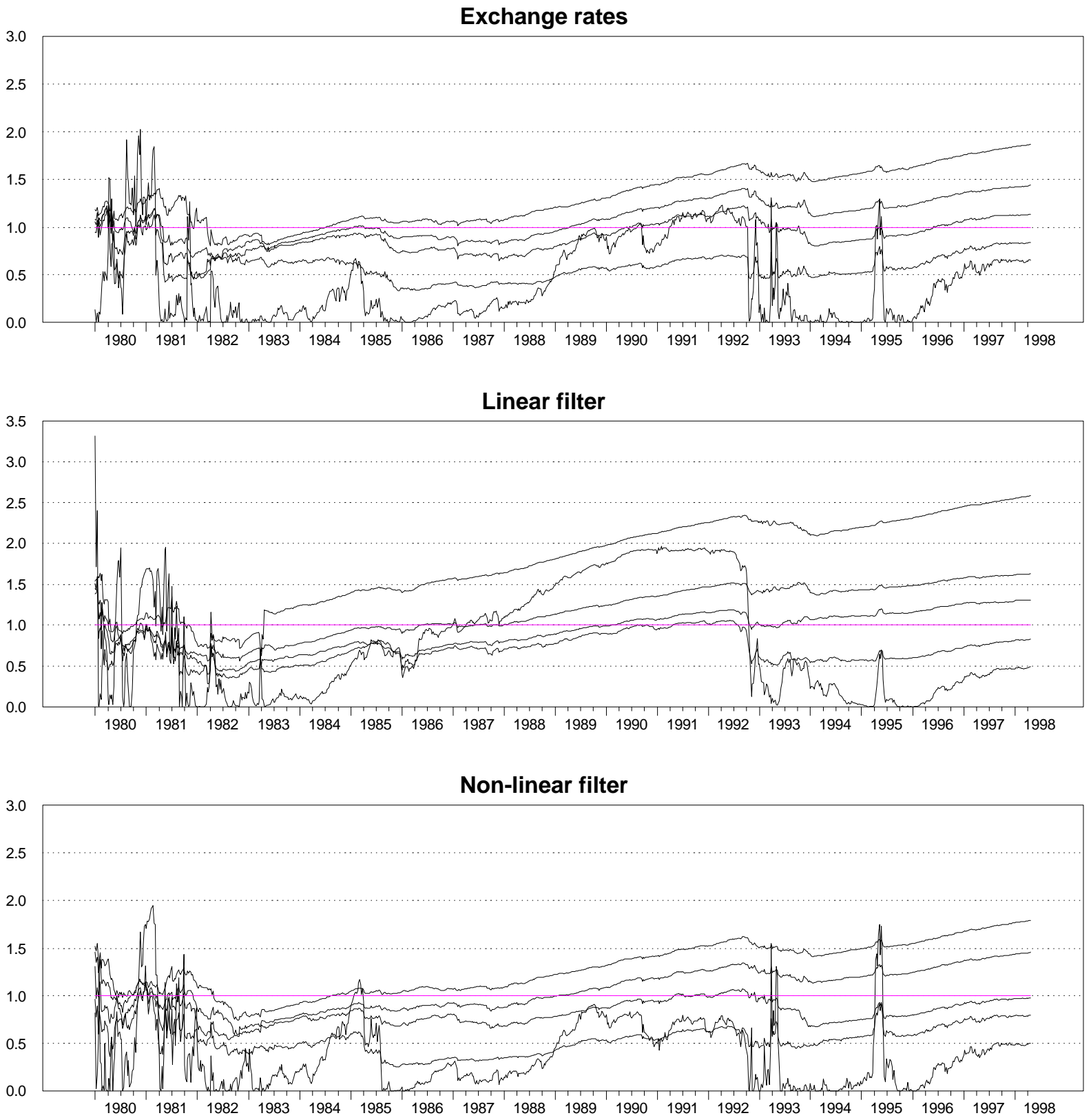
Figure 2: Rolling cointegration tests. Window-size $=3$ years

The figures show rolling cointegration tests scaled by their critical values (at 90 percentage confidence level) from VAR models containing respectively five ERM exchange rates, five shadow exchange rates from linear filters, and five shadow exchange rates from non-linear filters. A series above "one" suggests rejection of the hypothesis of respectively $r \leq i, i=$ $0,1,2,3,4$. For ease of presentation, the specific filters used to extract the underlying time series are not pointed out in the graphs.
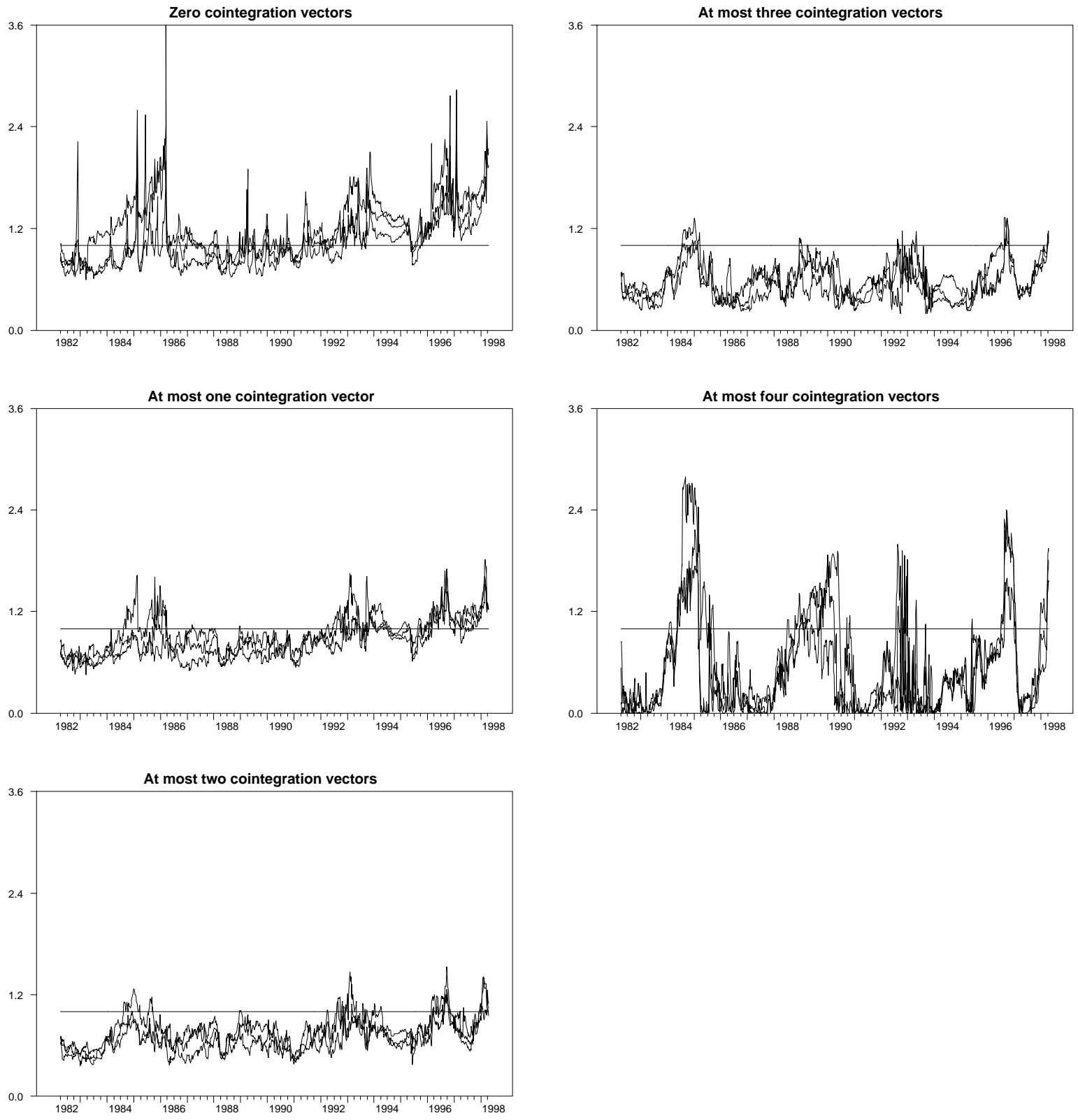
Figure 3: Phases of degree of convergence in the ERM

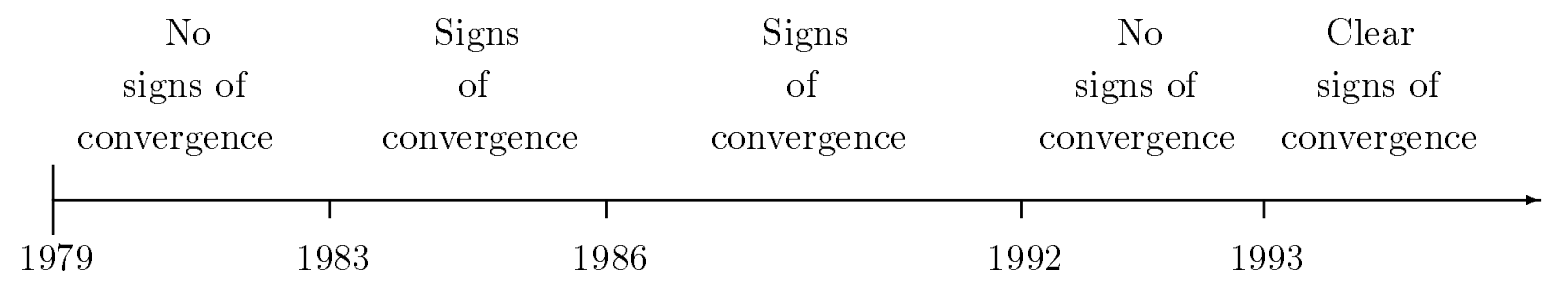


Figure 4: Recursive Dickey-Fuller tests

The figures show recursive Dickey-Fuller tests scaled by their critical values (at 90 percentage confidence level). The period March 13, 1979 to January 1, 1980 is reserved for initial estimates. A series below "one" suggests that the corresponding time series cannot be rejected as a non-stationary series. In the graphs, it is indicated whether the tests are based on shadow exchange rates extracted through the linear filter (1-fil), the non-linear filter (nl-fil), or the linear filter with $\delta=0$ (exh).
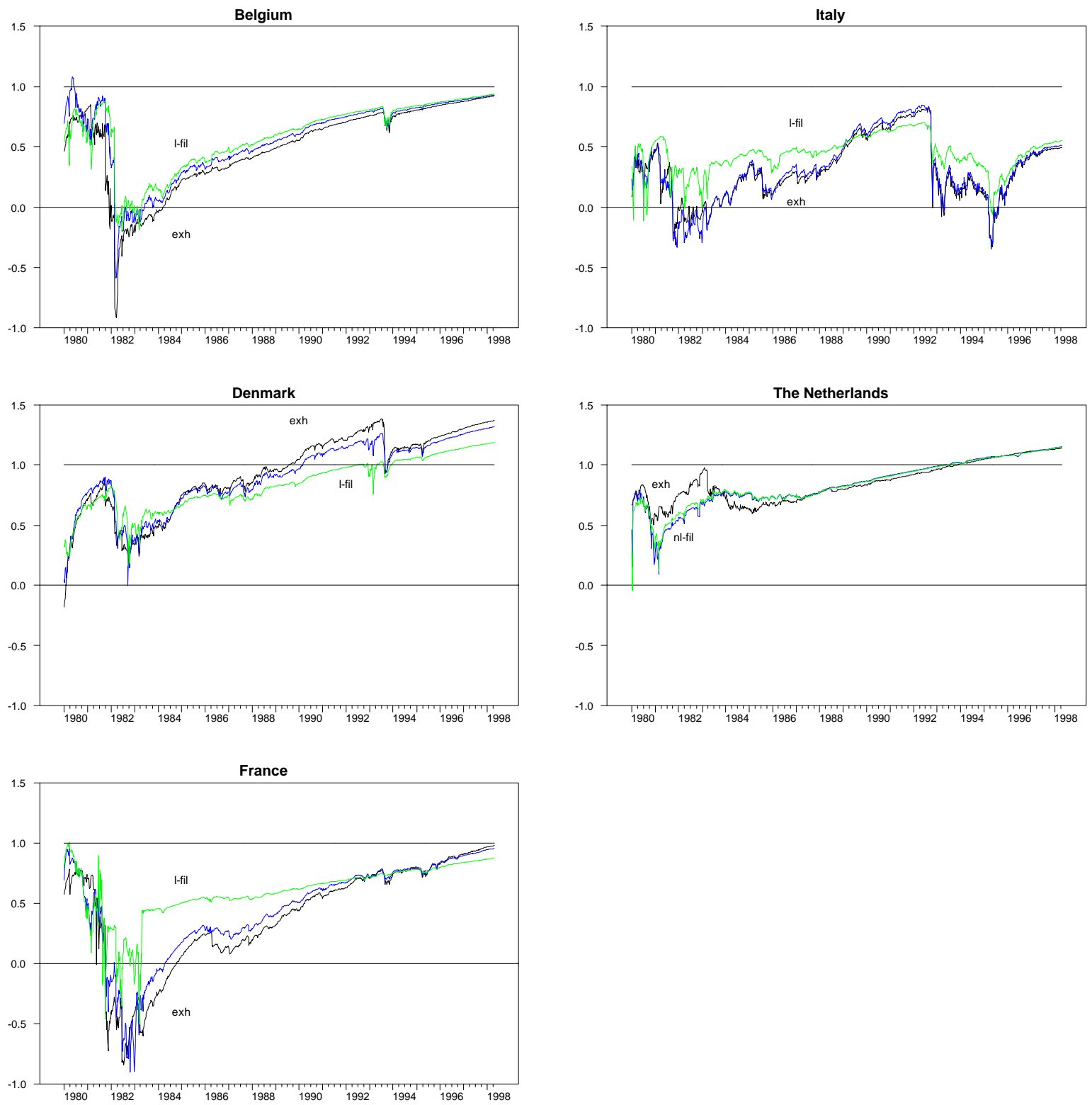